\title{
Valoración del papel del ecodoppler duplex color y la angiorresonancia magnética nuclear en el diagnóstico de la arteritis de Takayasu
}

\author{
M. LABIÓS GÓMEZ, F. G. BOTELLA, Mª . V. GUIRAL OLIVÁN, Mª D. S. MARTÍNEZ \\ APARICIO
}

Servicio de Medicina Interna. Hospital Clínico Universitario de Valencia

\begin{abstract}
EVALUATION OF THE ROLE OF COLOUR DOPPLER DUPLEX IMAGING ULTRASOUND AND MAGNETIC NUCLEAR ANGIORESO -
\end{abstract} NANCE IN DIAGNOSING TAKAYASU ARTERITIS

\begin{abstract}
RESUMEN
La arteritis de Takayasu (AT) es un proceso inflamatorio crónico de etiología desconocida que afecta a la aorta y sus ramas principales, lo que provoca oclusiones, estenosis o dilataciones en los vasos afectados. Presentamos un caso de AT en una mujer joven estudiada por ecodoppler duplex color y confirmada por angiorresonancia magnética nuclear (ARMN). Discutimos el papel de estas dos exploraciones en la detección y el seguimiento de la enfermedad. Debido a que su expresión y edad de presentación puede variar según las diferentes áreas geográficas afectadas, hacemos especial hincapié en los nuevos criterios diagnósticos para esta entidad, basados en los hallazgos angiográficos obtenidos.
\end{abstract}

PALABRAS CLAVE: Arteritis de Takayasu. Ecodoppler duplex color. Angiorresonancia magnética nuclear.

\begin{abstract}
Takayasu arteritis (TA) is a chronic inflammatory process of unknown etiology which affects the aorta and its main branches causing occlusion, stenosis or dilation of the vessels concerned. A case of TA in a young woman is presented, who underwent colour doppler duplex ultrasound image study and whose diagnosis was confirmed by magnetic nuclear angioresonance $(M N A R)$. The role of these two explorations in the detection and follow-up of this condition is discussed. Due to the fact that the expression and age at presentation may vary according to the different geographical areas affected, we would especially underline the new diagnostic criteria for this entity and based on the angiography findings obtained.
\end{abstract}

KEY WORDS: Takayasu arteritis. Colour doppler duplex ultrasound. Magnetic nuclear angioresonance.

Labiós Gomez M, Botella FG, Guiral Oliván $M^{a} V$, Martínez Aparicio $M^{a} D S$. Valoración del papel del ecodoppler duplex color y la angiorre sonancia magnética nuclear en el diagnóstico de la arteritis de Takayasu. An Med Interna (Madrid) 2002; 19: 31-34.

\section{INTRODUCCIÓN}

La arteritis de Takayasu es una arteriopatía inflamatoria crónica de etiología desconocida que afecta a los grandes vasos como la aorta y sus ramas principales, y, en menor extensión, a las arterias pulmonares (1). Se caracteriza por una inflamación crónica inespecífica de todas las capas arteriales, lo que provoca estenosis, oclusiones, dilataciones, o formaciones aneurismáticas. Por ello, las manifestaciones clínicas varían ampliamente al depender de los vasos afectados, lo que hace difícil su diagnóstico precoz (2).

Tradicionalmente se consideraba que esta enfermedad quedaba restringida a un ámbito geográfico determinado,- Asia y Sudamérica-, pero, en la actualidad, se confirma plenamente su presencia en cualquier área (3). Sin embargo, sí parece existir una relación entre el modo de manifestarse la enfermedad y el lugar en el que lo hace. Aunque esta afecte predominantemente a mujeres jóvenes, los últimos estudios demuestran que la afección presenta diferencias tanto en la forma de expresión como en la edad de los pacientes según el lugar de origen de los mis- mos (4). Esto ha llevado a diversos autores a desarrollar nuevos criterios diagnósticos para esta entidad basados en los hallazgos angiográficos obtenidos (5).

Su diagnóstico, generalmente, es confirmado por la angiografía de contraste, considerada el estándar de referencia (6). Sin embargo, esta técnica únicamente evalúa la luz y la extensión de los vasos lesionados, pero, no proporciona información sobre el estado de sus paredes. Por todo ello, no se puede considerar el método diagnóstico ideal, ya que no permite el adecuado seguimiento de la enfermedad ni la valoración de los resultados obtenidos con los diferentes tratamientos empleados $(6,7)$.

En los últimos años, se han generalizado las técnicas no invasoras del diagnóstico por la imagen en la evaluación de los pacientes con AT (6). La ultrasonografía ha demostrado una alta sensibilidad y especificidad para las lesiones oclusivas o estenóticas, cuando se la compara con la angiografía ya que además detecta cambios hemodinámicos y la composición estructural de las capas arteriales estudiadas $(8,9)$. La ARMN, al contrario que la tomografía axial computadorizada (TAC), evita el riesgo de exposición a las radiaciones repetidas, diferencia un proceso

Trabajo aceptado: 24 de Mayo de 2000

Correspondencia: F. G. Botella. C/Conde Altea 49 - pta 3 ${ }^{\text {a }} 46005$ Valencia. 
inflamatorio agudo de una fibrosis, y proporciona imágenes adecuadas en cualquier plano deseado $(10,11)$.

Describimos un caso de AT en una mujer de treinta y tres años, en fase crónica de la enfermedad. Destacamos los nuevos criterios diagnósticos, así como el papel potencial del ecodoppler duplex color y de la ARMN como métodos no invasores para el diagnóstico y monitorización de las arteritis de los grandes vasos.

\section{CASO APORTADO}

Mujer de treinta y tres años, fumadora, con antecedentes de hipertensión arterial (HTA) desde hacía cinco años, tratada con antagonistas del calcio e inhibidores de la enzima convertidora (IECAS), que consultó por cefalea y claudicación de los miembros inferiores de un año de evolución. En la exploración física se encontró una tensión arterial (TA) de 190/120 mm Hg en ambos miembros superiores, con unos pulsos periféricos conservados y simétricos en las cuatro extremidades. Realizada una monitorización ambulatoria de presión arterial, se confirmaron unas TA medias, diurnas y nocturnas, de $154 / 98 \mathrm{~mm} \mathrm{Hg}$ y $152 / 90 \mathrm{~mm} \mathrm{Hg}$, respectivamente, con una TA media a las veinticuatro horas de 153/96 mm Hg.

Los análisis básicos fueron normales, incluido hemograma, VSG, química hemática, orina estandar, estudios de coagulación, marcadores tumorales, sistema de complemento, proteina $\mathrm{C}$ reactiva, ASLO, pruebas luéticas, anticuerpos antinucleares, anti- DNA, anti $\mathrm{Sm}$, anticardiolipina, antifosfatidil serina, y anticoagulante circulante (inhibidor del tiempo de tromboplastina, reptilasa, y cefalina diluidos). La radiografía torácica y el ECG también fueron normales.

A la paciente se le efectuó un ecodoppler duplex color abdominal (Fig. 1), que reveló una marcada alteración del espectro de flujos en las arterias interlobares del riñón derecho, apreciándose una morfología de onda "tardus-parvus", altamente sugestiva de estenosis de la arteria renal derecha. Así mismo, tanto su volumen como el grosor de la cortical presentaban una marcada disminución respecto al riñón izquierdo.

Efectuada la ARMN del arco aórtico y sus ramas principales,- vasos pulmonares, cervicales, cerebrales, aorta torácica y abdominal, e iliacofemoropopliteas-, con un equipo de 1,5 TESLAS y con técnica de 2DTOF/ SPGR, para obtener secuencias angiográficas tras la inyección de un bolus de gadolinio con técnica de 3DMRDSA, muestra (Fig. 2A) una oclusión distal de la aorta abdominal inmediatamente posterior a la salida de las arterias renales, con engrosamiento de su pared.Las arterias femorales comunes se rellenan a partir de las ramas subcutáneas de las arterias intercostales, que se dirigen hacia el canal inguinal. Este hallazgo se visualiza tanto en las secuencias localizadoras como en las imágenes del 3DMRDSA.Las arterias femorales comunes y superficiales, poplíteas y tibiales eran normales en ambos miembros inferiores. Existe también una estenosis severa de la arteria renal derecha (Fig. 2B), que comporta una importante disminución del tamaño del riñón derecho, si se compara con el izquierdo, que presenta una nefromegalia compensadora con un retorno venoso rápido e importante.La salida de la arteria mesentérica superior (Fig. 2C), se visualiza muy irregular en su origen, $\mathrm{y}$ con aspecto arrosariado, lo que indica una estenosis de la misma.

Siguiendo las clasificaciones tradicionales, y en función del área aórtica afectada y las pruebas biológicas realizadas, nuestra enferma fue diagnosticada de arteritis de Takayasu tipo II en fase crónica.

\section{DISCUSIÓN}

La AT se caracteriza por una afectación predominantemente aórtica, lo que clásicamente y en función de los hallazgos angiográficos, ha llevado a clasificarla en cuatro tipos diferentes $(6,12)$ : órticas.

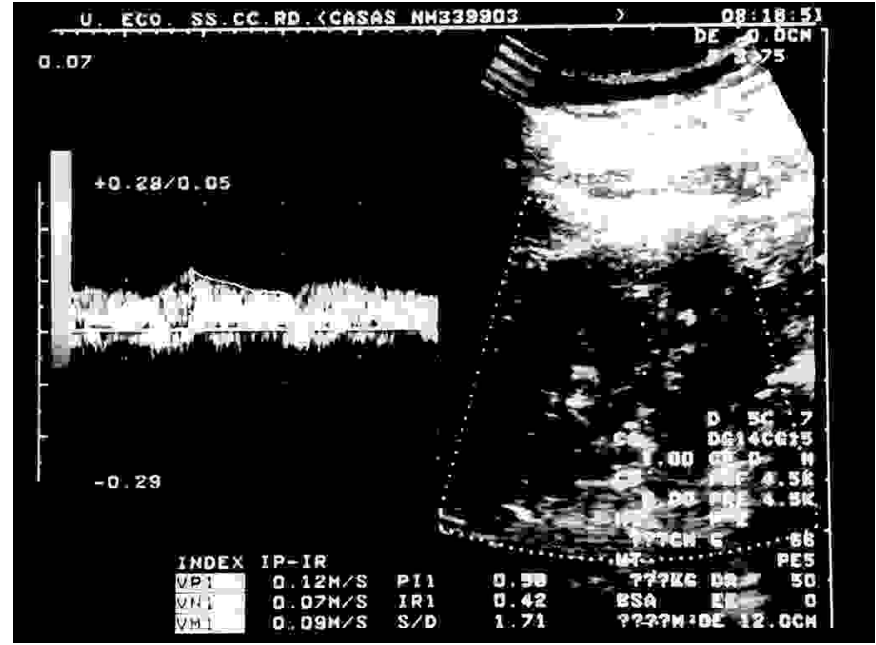

Fig. 1. Ecodoppler duplex color: corte sagital del riñón derecho con marcada alteración del espectro de flujos en sus arterias interlobares. Se aprecia una morfología de onda "tardus-parvus", altamente sugestiva de estenosis de la arteria renal derecha. Tanto su volumen como el grosor de su cortical presentan una marcada disminución respecto al riñón izquierdo.

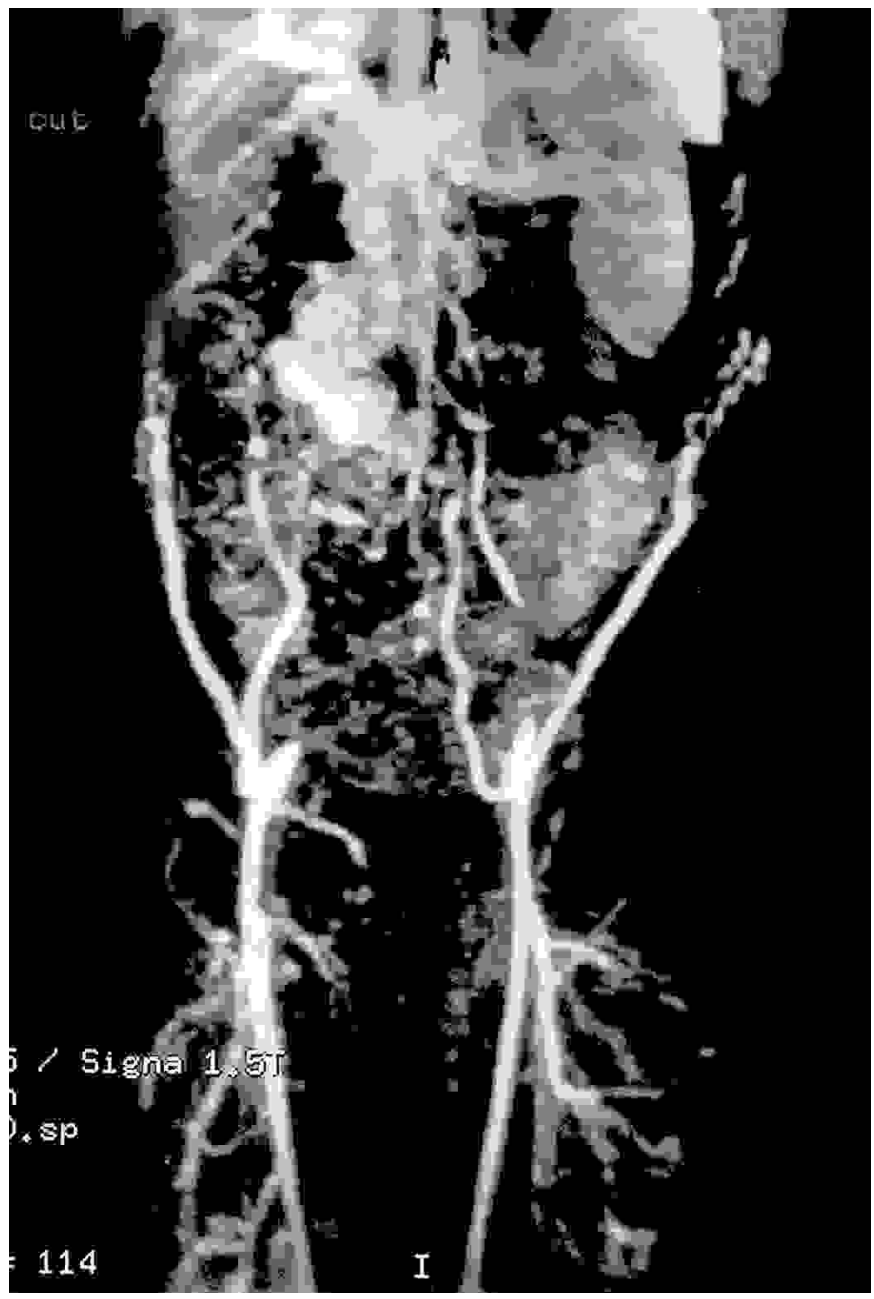

Fig. 2a. Corte coronal ARM N(2TOF/ SPGR), que muestra una oclusión distal de la aorta abdominal inmediatamente posterior a la salida de las arterias renales, con engrosami ento de su pared. Las arterias femorales comunes se rellenan a partir de las ramas subcutáneas de las arterias intercostales, que se dirigen hacia el canal inguinal. 


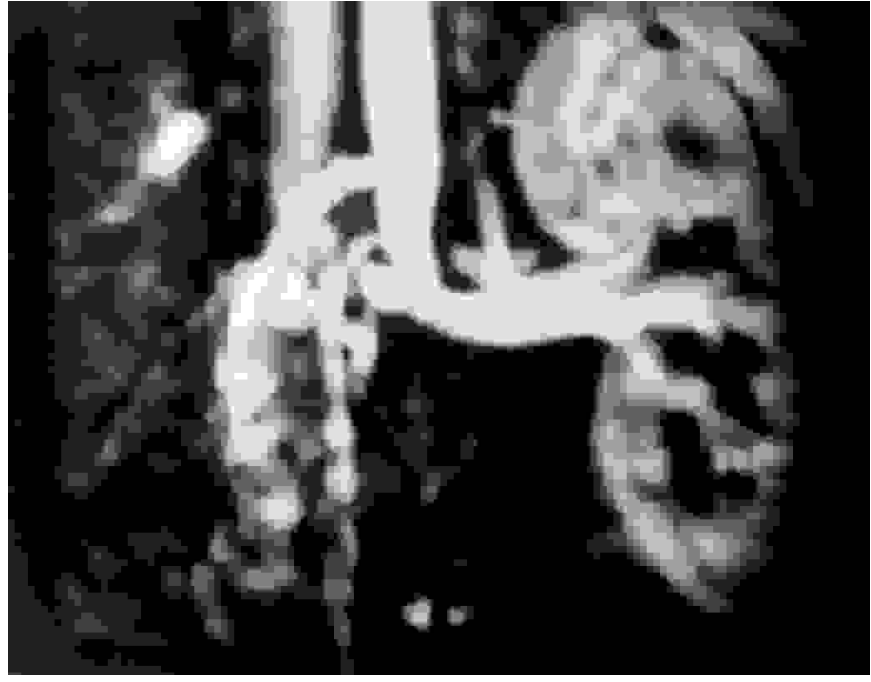

Fig. 2b. Corte coronal ARM N(2TOF/SPGR), en el que se observa una estenosis severa de la arteria renal derecha, que conlleva una importante disminución del tamaño del riñón derecho.

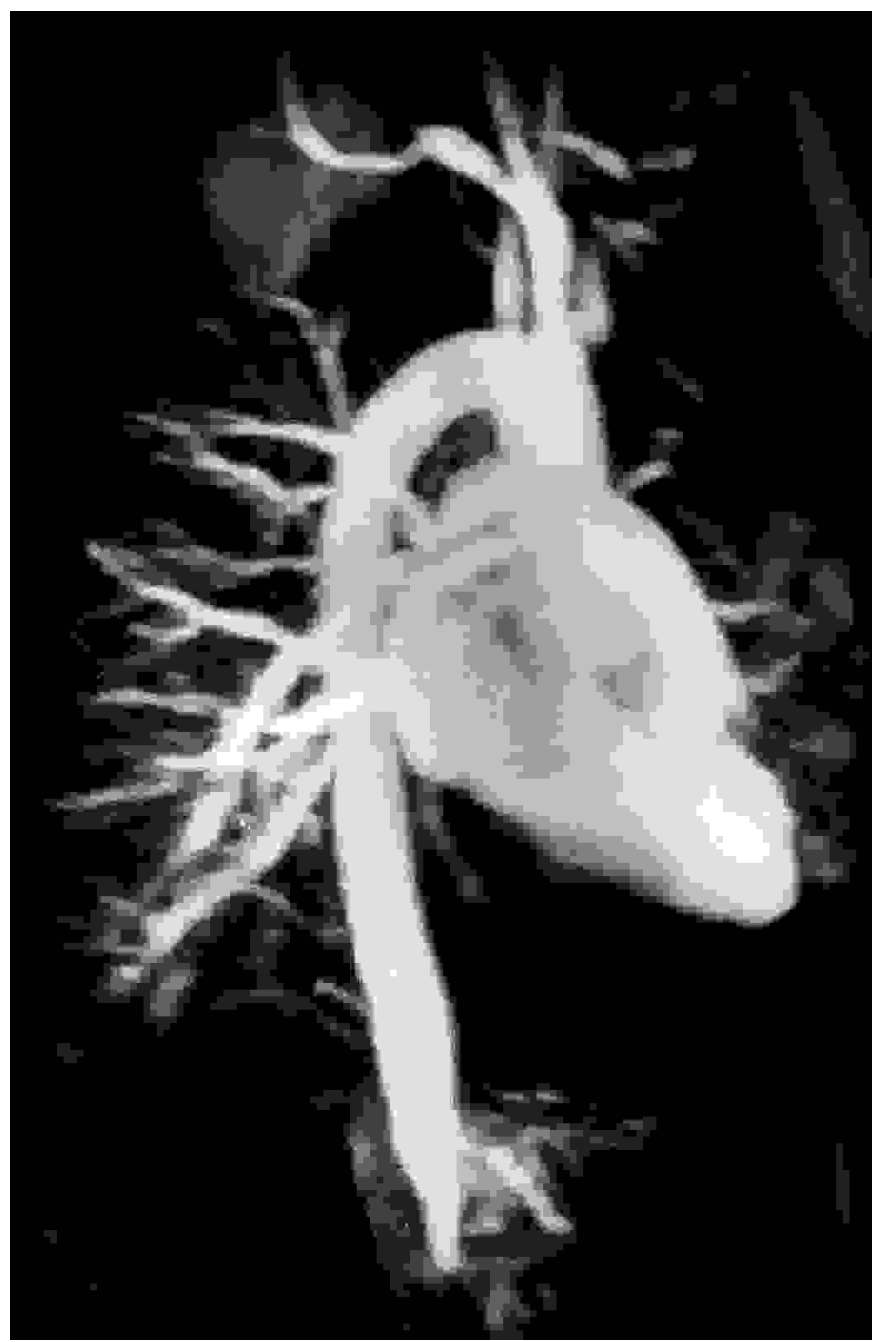

Fig. 2c. Corte sagital ARM N(2TOF/SPGR), que muestra una arteria mesentérica superior irregular en su origen, y con aspecto arrosariado, indicativo de estenosis de la misma (flecha).
-Tipo II: afecta a la aorta abdominal y sus ramas. A este tipo correspondería nuestra paciente, que presentaba unos pulsos arteriales conservados y simétricos en las extremidades inferiores gracias a que las arterias femorales comunes se rellenaban a partir de las ramas subcutáneas de las arterias intercostales.

-Tipo III: mezcla del tipo I y II.

-Tipo IV: caracterizado por la afectación de la arteria pulmonar.

Sin embargo, en los últimos años se ha puesto de manifiesto que la edad y la expresión de la enfermedad puede variar en las diferentes áreas geográficas afectadas (4). Por ejemplo, la fuerte predominancia femenina observada en Japón puede no ser tan pronunciada en otros paises. Aunque en la mayoría de los casos, la AT es diagnosticada entre la segunda y la tercera década de la vida, informes recientes demuestran que el $13 \%$ y el $15 \%$ respectivamente de los pacientes diagnosticados en Japón y EE.UU. sobrepasan los 40 años $(3,13)$. Por otra parte, la clásica afectación del arco aórtico y sus ramas supraaórticas, descrita en las series japonesas, pueden ser menos frecuentes en la India, Tailandia y Sudamérica, donde la afectación de las arterias torácicas y abdominales es más común $(3,5)$. Consecuentemente, la regurgitación aórtica, el vértigo y la ausencia de pulso en las extremidades superiores es más frecuente en Japón. Por el contrario, en el sudeste asiático, la hipertensión renovascular lleva al diagnóstico de la enfermedad en una proporción substancial de individuos (14). Por todo ello, distintos grupos de investigadores han propuesto una nueva clasificación de la AT en un intento de definir mejor a este tipo de pacientes $(5,14)$.

-Tipo I: localizado en las ramas supraaórticas del arco aórtico.

—Tipo IIa: afecta a la aorta ascendente y al arco aórtico con sus ramas.

-TipoIIb: afectación de la aorta ascendente, arco aórtico con sus ramas y aorta torácica descendente.

-Tipo III: comprende la aorta torácica descendente, abdominal y/o arterias renales.

-Tipo IV: afecta la aorta abdominal y/o las arterias renales, al que pertenecería nuestra enferma.

-Tipo V: combina los hallazgos del tipo IIb y IV. La afectación de las arterias coronarias o pulmonares se designarían como $\mathrm{C}+$, o $\mathrm{P}+$.

Las típicas manifestaciones angiográficas de la AT en su fase crónica incluyen las estenosis y las oclusiones, como en nuestro caso, o las dilataciones aneurismáticas de la aorta y sus ramas, así como de la arteria pulmonar $(3,15)$. Sin embargo, al valorar únicamente la luz del vaso, la aortografía es incapaz de distinguir entre una estenosis originada por una inflamación activa, de una fibrosis $(6,7)$. Con este método diagnóstico no se manifiestan los cambios inflamatorios de la adventicia y de la media expresados por el engrosamiento de las paredes arteriales, que son los rasgos mas significativos en la fase aguda de la enfermedad (11). Las principales limitaciones de los estudios angiográficos repetidos en la monitorización de la progresión de la enfermedad son las radiaciones tóxicas acumulativas y el riesgo, poco frecuente pero posible, de provocar infecciones, hemorragias, roturas de aneurismas y complicaciones isquémicas (7).

Todo ello, ha acelerado la introducción de técnicas diagnósticas no invasoras como el ecodoppler duplex color y la ARMN en la evaluación de esta enfermedad $(8,11)$. En su detección, la ecografía con sus transductores de alta resolución tiene ciertas 
ventajas sobre la angiografía.En primer lugar, el engrosamiento de la íntima y de la media, característica precoz de la AT puede ser detectado fácilmente (8). No se dio en nuestra paciente debido, probablemente, a la profundidad en la que se encontraban en el parénquima renal las arterias interlobares. En segundo lugar, porque es superior a la angiografía en la detección y cuantificación de los cambios hemodinámicos, incluida la alteración del espectro de flujos, lo que probablemente refleje una mala elasticidad de la pared arterial secundaria a la inflamación $(8,16)$, o, como en nuestro caso, a la fibrosis. En tercer lugar, porque es un método no invasor, eficiente, accesible, fiable y adecuado para el seguimiento a largo plazo de la enfermedad (8). También tiene sus limitaciones, ya que para detectar una estenosis, esta debe ser superior al $50 \%$ para provocar un cambio en el patrón del flujo arterial detectado (17), lo que posiblemente explique el porqué no identificó directamente la estenosis de la arteria renal derecha en nuestra paciente. No hay que olvidar tampoco que en estas arterias el ecodoppler únicamente proporciona una evidencia indirecta de la presencia de lesiones (18). La aorta torácica y la arteria pulmonar son difíciles de ver adecuadamente, debido al parénquima pulmonar circundante. Ocasionalmente, una o más de las ramas principales de la aorta abdominal pueden ser difíciles o imposibles de evaluar debido al gas intraabdominal, o a la presencia de tejido adiposo (16). En nuestra paciente, tampoco detectó la afectación de la arteria mesentérica superior. Incluso, las estenosis mínimas en las ramificaciones de los vasos principales y las sutiles irregularidades angiográficas pueden no ser detectadas por el ecodoppler color (16).

La TAC y la ARMN, han sido utilizadas en la valoración de los pacientes con sospecha de AT $(19,20)$. Ambas, generalmente, identifican los cambios luminales experimentados por los grandes vasos; estenosis, oclusiones, dilataciones aneurismáticas, engrosamiento de sus paredes,calcificaciones y trombos murales. Sin embargo, la TAC presenta una serie de inconvenientes como son, el proporcionar imágenes únicamente en un plano transversal, el necesitar contrastes iodados y el riesgo de exposición a las radiaciones repetidas (11). La aparición del
TAC helicoidal ha paliado, en parte, estas limitaciones al permitir obtener tanto una información angiográfica convencional, como dinámica de los cambios murales sufridos por la aorta y las arterias pulmonares (19). Por el contrario, la ARMN, como en nuestro caso, puede proporcionar una visión rápida y multiplanar de estas arterias lo que es de extraordinaria utilidad a la hora de analizar los cambios intraluminales de la aorta orientada verticalmente, así como para la valoración del grado y extensión de la arteritis en la fase oclusiva última de la enfermedad (11).

La ARMN revela que las estenosis segmentarias extensas se desarrollan en la aorta torácica descendente y abdominal, como en nuestra enferma, pero nunca en la aorta ascendente y en el arco aórtico (11). Las irregularidades y el engrosamiento de la pared aórtica son frecuentemente detectadas, sobre todo en la aorta torácica descendente. La ARMN también puede manifestar lesiones arteriales pulmonares, que son específicas de la AT y no son vistas en los casos de aterosclerosis ni en otras arteritis (11). Esta prueba al demostrar las lesiones de la circulación sistémica y pulmonar sin necesidad de contraste, además de no provocar reacciones adversas, podría convertirse en un método seguro, tanto de diagnóstico como de seguimiento de la enfermedad (11). La ARMN, presenta algunas limitaciones, principalmente la de no mostrar con la suficiente nitidez las lesiones de las ramas distales del arco aórtico, lo que hace muy difícil su evaluación $(11,21)$. De todas formas, la angiografía convencional puede ser necesaria, todavía, en los pacientes que al ser diagnosticados por primera vez se encuentran en fase oclusiva última. Ya que proporciona información adicional sobre el grado y extensión de la arteritis (11).

Por todo lo expuesto, pensamos que si el ecodoppler duplex color y la ARMN consiguen superar las dificultades técnicas que les impiden visulizar adecuadamente las lesiones de las ramas distales de los grandes vasos, pueden convertirse en una herramienta eficiente y fiable en el caso del ecodoppler y un procedimiento con alto grado de sensibilidad en el caso de la ARMN, tanto para la caracterización como para el seguimiento de los cambios vasculares en las arteritis de los grandes vasos.

\section{Bibliografía}

1. Yamato M, Lecky J, Hiramatsu K, Kohda E. Takayasu arteritis: radiographic and angiographic findings in 59 patients. Radiology 1986; 161: 329-334.

2. Raninen RO, Kupari MM, Pamilo MS, Pajari RI, Poutanen VP, Hekali PE. Arterial Wall thickness measurements by B mode ultrasonography in patients with Takayasu`s arteritis. Ann Rheum Dis 1996; 55: 461-465.

3. Kerr GS. Takayasu`s arteritis. Curr Opin Rheumatol 1994; 6: 32-38.

4. Numano F. Differences in clinical presentation and outcome in different countries.Takayasu`s arteritis. Curr Opin Rheumatol 1997; 9: 12-15.

5. Moriwaki R, Noda M, Yajima M, Sharma BK, Numano F. Clinical manifestations of Takayasu arteritis in India and Japan. New classification of angiographic findings. Angiology 1997; 48: 369-379.

6. Kerr GS. Takayasu`s arteritis. Rheum Dis Clin N Am 1995; 21: 1041-1058.

7. Cid Mc, Font C, Coll-Vinent B, Gran JM. Large vessel vasculitides. Curr Opin Rheumatol 1998; 10: 18-28.

8. Sun Y, Yip PK, Jeng JS, Hwang BS, Lin WH. Ultrasonographic study and long-term follow-up of Takayasu`s arteritis. Stroke 1996; 27: 2178-2182.

9. Antoniou A, Vlahos L, Mourikis D. Abdominal Takayasu`s arteritis: imaging with color duplex sonography. Eur Radiol 1998; 8: 547-549.

10. Yamada I, Numano F, Suzuki S. Takayasu arteritis: Evaluation with MR Imaging. Radiology 1993; 188: 89-94.

11. Matsunaga N, Hayashi K, Sakamoto I, Matsuoka Y, Ogawa Y, Honjo K, Takano K . Takayasu arteritis: MR manifestations and diagnosis of acute and chronic phase. JMRI 1998; 8: 406-414.

12. Jung EM, Bauman R, Ranh G, Müller-Höcker J. Unusual presentation of Takayasu arteritis with cardiac involvement and irritation of juvenile arterios- clerosis. Angiology 1996; 47: 399-406.

13. Sharma BK Jain S, Suri S, Numano F. Diagnostic criteria for Takayasu arteritis. Int J Cardiol 1996; 54 (suppl ): S 141-S 147.

14. Hata A, Noda M, Moriwaki R, Numano F. Angiographic findings of Takayasu arteritis: new classification. Int J Cardiol 1996; 54 (suppl): S 155-S 163.

15. Hoffman GS. Takayasu arteritis: lessons from the American National Institutes of Health experience. Int J Cardiol 1996; 54 (suppl): S 99-S 102.

16. Buckley A, Southwood T, Culham G, Nadel H, Malleson P, Petty R. The role of ultrasound in evaluation of Takayasu`s arteritis. J Rheumatol 1991; 18: 1073-1080.

17. Nicholls SC, Kholer TR, Martin RL, Neff R, Phillips DJ, Strandess DE. Diastolic flow as a predictor of arterial stenosis. J Vasc Surg 1986; 3: 498501.

18. Gilfeather M, Hyo-Chun Y, Evans S, et al. Renal artery stenosis: evaluation with conventional angiography versus gadolinium-enhaced MR angiography. Radiology 1999; 210: 367-372.

19. Park JH, Chung JW, Im JG, et al. Takayasu arteritis: evaluation of mural changes in the aorta and pulmonary artery with CT angiography. Radiology 1995; 196: 89-93.

20. Sharma S, Sharma S, Taneja K, Gupta AK, Rajani M. Morphologic mural changes in the aorta revealed by CT in patients with nonspecific aortoarteritis (Takayasu's Arteritis). AJR 1996; 167: 1321-1325.

21. Miller DL, Reinig JW, Volkman DJ. Vascular imaging with MRI: inadequacy in Takayasu`s arteritis compared with angiography. Am J Roentgenol 1986; 146: 949-954. 